\title{
COLD ISCHEMIA-INDUCED DAMAGE TO VASCULAR ENDOTHELIUM RESULTS IN PERMEABILITY ALTERATIONS IN TRANSPLANTED LUNGS
}

M. A. Hidalgo, MD ${ }^{\mathrm{a}, \mathrm{b}}$

K. A. Shah, MRCPath ${ }^{\mathrm{a}}$

B. J. Fuller, $\mathrm{PhD}^{\mathrm{b}}$

C. J. Green, PhD, DSc(Med),

FRCVS, FRCPath, FRCS(Hon), Acad $^{\text {n } U k r, ~ A c a d S c i ~}{ }^{a}$
Despite suggestions of a connection between endothelial damage and permeability alterations after ischemia and reperfusion in pulmonary tissue undergoing transplantation, no direct correlation between vascular endothelial discontinuity and parenchymal edema has yet been shown. Methods: Forty-two rat lungs were harvested and stored for 48 or 72 hours under hypothermic and ischemic conditions. Stored pulmonary tissue was studied before transplantation and 5 minutes or 24 hours after transplantation by light microscopy and scanning electron microscopy of arterial vascular endothelium. Results: Stored lungs not subjected to revascularization showed moderate perivascular edema, with small intercellular gaps in endothelial monolayers. Five minutes after transplantation, pulmonary tissue appeared congested, with perivascular and alveolar edema. Examination of vascular endothelium by scanning electron microscopy showed detachment of endothelial cells. Twenty-four hours after transplantation, edema, hemorrhage, and vascular congestion were found in all specimens. Arterial vascular endothelium showed weak intercellular connections, numerous intercellular gaps, and widespread cell detachment. Bronchial epithelial cells appeared damaged after storage, with loss of cilia, blebbing of apical cytoplasm, and cellular rounding. These changes were maintained 5 minutes after transplantation but appeared totally reversed after 24 hours in specimens stored 48 hours, whereas bronchial denudation was observed in 72-hour stored lungs. Statistically significant positive correlations (Kendall $p<0.001$ ) between revascularization time and alveolar edema and hemorrhage were found for both storage periods. Conclusion: The results from this study demonstrate correlation between loss of endothelial monolayer continuity and histologic evidence of vascular permeability increases in pulmonary tissue before and after lung transplantation. (J Thorac Cardiovasc Surg 1996;112:1027-35)
From the Department of Surgical Research, Northwick Park Institute for Medical Research, Northwick Park Hospital, Harrow, Middlesex, ${ }^{a}$ and the University Department of Surgery, Royal Free Hospital School of Medicine, Hampstead, London, ${ }^{\mathrm{b}}$ United Kingdom.

This work was partially funded by a grant from the Basque Country Government, Programa de Formación de Investigadores, Department of Education, Universities and Research.

Received for publication Sept. 26, 1995; revisions requested Jan. 17, 1996; revisions received June 13, 1996; accepted for publication June 17, 1996.

Address for reprints: K. A. Shah, Department of Surgical Research, Northwick Park Institute for Medical Research, Northwick Park Hospital, Watford Road, Harrow, Middlesex HA1 3UJ, United Kingdom.

Copyright (c) 1996 by Mosby-Year Book, Inc.

$0022-5223 / 96 \$ 5.00+0 \quad \mathbf{1 2 / 1 / 7 5 8 9 0}$
$\Lambda$ single flush through the vascular space of solid Aorgans with a specifically designed preservation solution followed by hypothermic storage is the method of choice in most clinical transplant units. ${ }^{1}$ The length of storage time that organs can tolerate without losing their viability varies from organ to organ. Lungs are among the most sensitive to hypothermic and ischemic injury, with only short safe storage times. ${ }^{2,3}$ Early and universal development of edema is the first indication of storage-induced damage after lungs have been transplanted. ${ }^{4-6}$

Loss of control of vascular permeability seems to be an inevitable consequence of extended pulmonary hypothermic storage. ${ }^{7}$ Under physiologic conditions, regulation of vascular permeability relies on functioning endothelium, and alterations in endothelial cell (EC) shape have marked effects on 

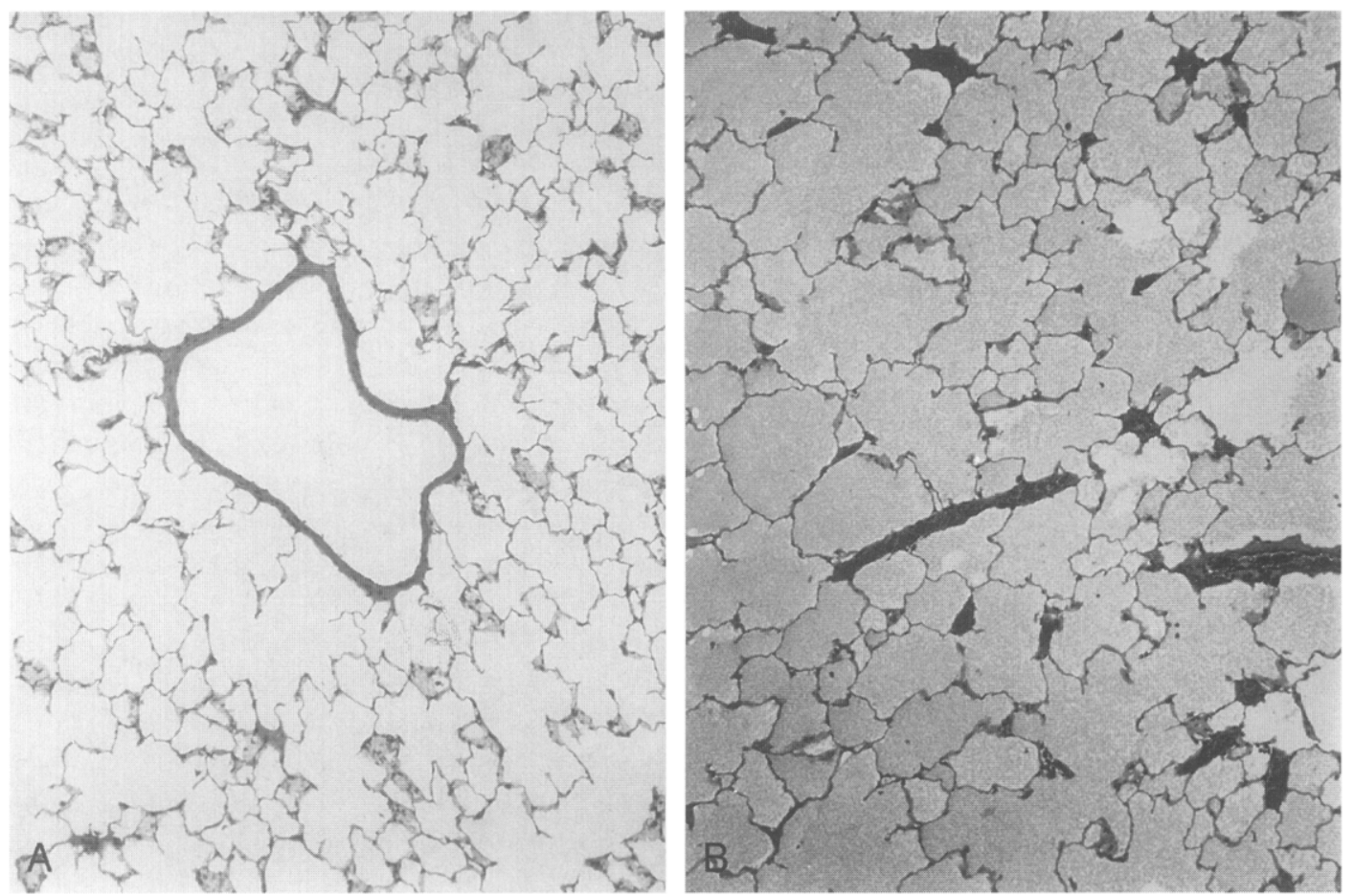

Fig. 1. A, Control: Normal rat lung tissue. B, Alveolar edema and congestion after 48 hours of storage and 5 minutes of revascularization. (Original magnifications $\times 63$.)

Table I. Composition of the modified University of Wisconsin solution (UW) used in this experiment

\begin{tabular}{lc}
\multicolumn{1}{c}{ Components } & $U W(\mathrm{mmol} / \mathrm{L})$ \\
\hline Lactobionic acid & 107.7 \\
Potassium hydroxide & 104.2 \\
Dipotassium hydrogen phosphate & 26 \\
Magnesium hydrogen phosphate & 21.8 \\
Glutathione & 3.1 \\
Sodium hydroxide & 38.5 \\
Raffinose pentahydrate & 31.3 \\
Allopurinol & 1 \\
Disodium phosphate & \\
Histidine & \\
Dextran 40 & \\
Phallacidin & \\
pH & 6.87 \\
Osmolarity & 305 \\
\hline
\end{tabular}

vascular permeability. ${ }^{8,9}$ Recent studies have found that ECs are highly sensitive to ischemia and reperfusion damage, being less resistant to storage than parenchymal cells in organs undergoing transplantation. ${ }^{10,11}$

Despite the growing evidence of EC involvement in alteration of vascular permeability ${ }^{12}$ and suggestions that the edema resulting from ischemia and reperfusion injury is due to EC injury, ${ }^{13}$ no direct correlation between vascular endothelial discontinuity and parenchymal edema has yet been proven in organs being transplanted. The aim of the current study was to correlate histologic edema with ultrastructural endothelial monolayer alterations before and after revascularization in pulmonary tissue stored under hypothermic and ischemic conditions. For that purpose an in vivo model of single lung transplantation in the rat was used. The preservation solution used was a modification of the clinically used University of Wisconsin solution, widely implemented in experimental and clinical transplantation., ${ }^{5,}$ Pulmonary tissue was evaluated immediately after two different storage periods ( 48 or 72 hours) and after 5 minutes or 24 hours of in vivo revascularization after transplantation. The selection of 48 and 72 hours of ischemia was based on our experience with this model, which showed that the cold ischemic tolerance time in rat lungs is considerably longer than that for human beings. ${ }^{4}$ Despite the fact that current clinical preservation is limited to 4 to 6 hours, most contemporary experimental studies involve long periods of ischemic storage. ${ }^{3}$ Our aim was thus to inflict serious endothelial damage to visualize morphologic changes that could be correlated with different degrees of parenchymal edema and hemorrhage. 

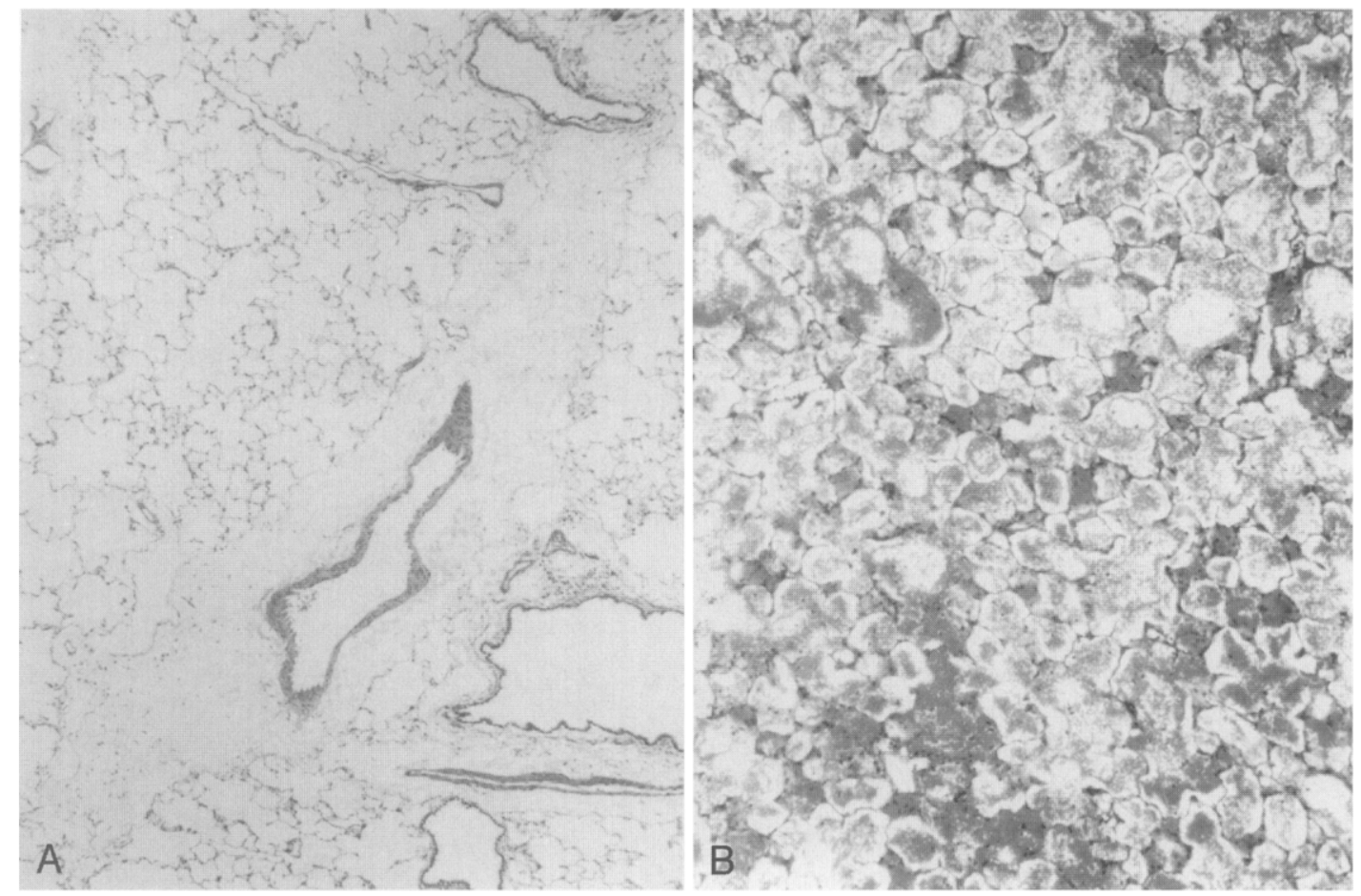

Fig. 2. A, Perivascular edema after 48 hours of storage in a nonreperfused specimen. $\mathbf{B}$, Alveolar hemorrhage after 72 hours of storage and 24 hours of revascularization. (Original magnifications: $\mathbf{A}, \times 40$; B, $\times 63$.)

\section{Materials and methods}

Materials. Lung transplants were performed in inbred female Lewis rats weighing $250 \mathrm{gm}$. Enflurane (Ethrane) for anesthesia was obtained from Abbott Laboratories Ltd., Queenborough, Kent, United Kingdom, and frusemide injection BP $(20 \mathrm{mg} / 2 \mathrm{ml})$ from Phoenix Pharmaceuticals, Gloucester, United Kingdom. Lactobionate-raffinose solution (modified University of Wisconsin solution) was prepared as described earlier, ${ }^{5}$ with the formulation presented in Table I. Electron microscopy reagents were obtained from Agar Scientific, Stansted, Essex, United Kingdom. The critical point dryer was from Polaron Equipment Ltd., Hertfordshire, United Kingdom. Electron microscopy samples were viewed with a JEOL 1200EX STEM microscope (JEOL USA, Inc., Peabody, Mass.).

\section{Methods}

Transplant procedure. All animals used in this experiment were treated in compliance with the Home Office regulations, Animals (Scientific Procedures) Act, 1986. The transplantation procedure is described in detail elsewhere. ${ }^{4}$ In brief, left lung lobes were harvested from fully anesthetized healthy donors, flushed with the modified University of Wisconsin solution (room temperature, 7 to $10 \mathrm{ml}$ ), and stored in that solution in a container surrounded by ice. The lungs were kept inflated and stored at $4^{\circ} \mathrm{C}$ for 48 or 72 hours. With the use of microsurgical instruments and techniques, stored lungs were orthotopically transplanted into genetically identical recipients af- ter removal of the native left lung. The recipient right lung was left in place, so that the animal would survive even without functional recovery of the transplanted lung.

Sample harvesting and processing. After predetermined periods of revascularization after transplantation, animals were put to death with an overdose of enflurane in an anesthetic chamber. Transplanted lungs were harvested via a midline sternal thoracotomy. Immediately after being harvested, lungs were fixed with 3\% glutaraldehyde in phosphate, $0.1 \mathrm{~mol} / \mathrm{L}$ ( 2 hours at room temperature), infused via the airway.

All lungs were processed for light microscopy and stained with hematoxylin and eosin. Three animals in each subgroup were randomly selected for scanning electron microscopic (SEM) studies. In those lungs, $2 \mathrm{~mm}^{2}$ blocks of full-thickness blood vessel wall were dissected and removed from the second intrapulmonary ramification of the main left bronchial artery with the use of microsurgical instruments and an operating microscope at high $(\times 16)$ magnification. Samples were washed (three times, 10 minutes) in phosphate buffer, $0.1 \mathrm{~mol} / \mathrm{L}$, fixed in $1 \%$ osmium tetroxide in phosphate buffer (60 minutes at room temperature), and washed again (10 minutes). Dehydration was accomplished by serial immersion of the samples for 10 minutes in $25 \%, 50 \%, 70 \%$, and $100 \%$ ethanol. Specimens were then critical-point dried and mounted on copper stubs with silver dag. They were gold coated in a sputter coater and kept in a vacuum desiccator until viewed with a JEOL electron microscope in the SEM mode. 

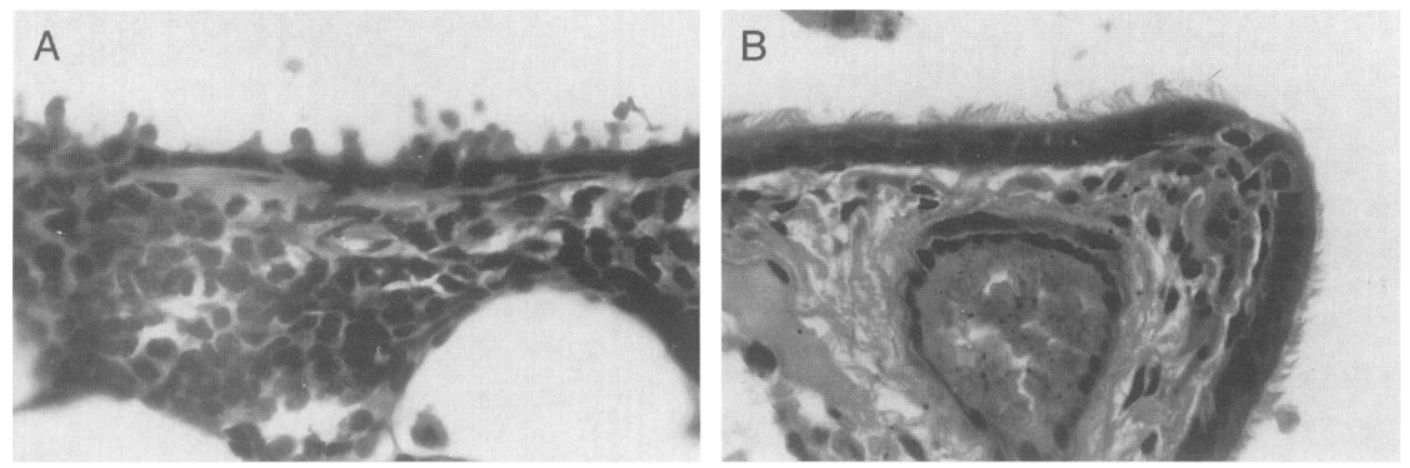

Fig. 3. A, Bronchial epithelial surface blebbing after 48 hours of storage. B, Totally restored bronchial epithelium after 24 hours of revascularization, which followed 48 hours of storage. (Original magnifications $\times 400$.)

Experimental protocol. A total of seven specimens were fixed without revascularization after each storage period (48 or 72 hours). The rest of the stored lungs were transplanted into recipients, which were put to death after 5 minutes or 24 hours.

The experimental protocol was as follows:

- Group 1: Controls. Group 1 included two subgroups: group $1 a$, nonstored, nontransplanted controls $(n=7)$; group $1 b$, nonstored, transplanted controls $(n=7)$.

- Group 2: Stored but nontransplanted lungs

- Group 3: Stored lungs revascularized for 5 minutes

- Group 4: Stored lungs revascularized for 24 hours

Groups 2, 3, and 4 consisted of two subgroups each: $a$, lungs stored 48 hours $(n=7) ; b$, lungs stored 72 hours $(n=7)$.

All subgroups consisted of seven specimens for light microscopic studies and three specimens for SEM studies. In group 4, animals that did not survive 24 hours of revascularization were disregarded. One additional transplant in subgroup 4a (48-hour storage and 24-hour revascularization) and three additional transplants in subgroup $4 \mathrm{~b}$ (72-hour storage and 24-hour revascularization) were carried out to reach the total of seven in this group.

Analysis of results

Histologic STUDY. All samples were analyzed blindly by one observer, and the analyses were subsequently corroborated by a second observer who was also blinded. Features studied were overall architectural preservation, edema (perivascular and alveolar), hemorrhage, congestion, and bronchial epithelial alterations.

All specimens were rated on a scale from 0 to $3: 0$ for normal histologic characteristics, 1 for mild alterations, 2 for moderate alterations, and 3 for severe changes. Intermediate changes were graded with an additional half grade (e.g., mild to moderate $=1.5$ ).

A Mann-Whitney $U$ test was performed to compare statistically the scores reached by each storage and revascularization period separately. Then, Kendall's rank correlation coefficient test was performed to relate the grade of histologic parameters with the revascularization periods within each storage time independently. For both tests, a $p$ value less than 0.05 was considered statistically significant.

SEM STUDY. Six different random fields of the luminal endothelial surface were photographed (final magnification $\times 1600$ ) for each specimen. A further three highmagnification micrographs (final magnification $\times 8000$ ) were taken from the same areas. All micrographs were analyzed in a blind manner by one observer, and the analyses were subsequently corroborated by a second observer. The features studied were (1) monolayer continuity, (2) EC morphologic characteristics, and (3) membrane surface alterations.

If the endothelium was $100 \%$ intact, the endothelial surface was scored as normal. In the experimental groups, samples were rated on an ascending scale of damage as follows: $0,90 \%$ or more of the endothelium was well preserved in all specimens; 1 , gaps or semidetached cells were found in $50 \%$ or more of the micrographs analyzed for each specimen; 2, cell detachment involving $50 \%$ or more of the endothelium was seen; and 3, more than $80 \%$ of the surface was denuded of ECs in one or more micrograph for each specimen.

Because of the small number of specimens in each individual group, we could not perform a nonparametric statistical analysis that could show any significant differences between each storage and revascularization period separately. Kendall's rank correlation coefficient test was used to relate the percentage of preserved endothelium with the revascularization periods within each storage time independently. Results with a $p$ value of less than 0.05 were considered statistically significant.

\section{Results}

Histologic analysis. Control specimens showed normal alveolar and bronchial structure with no evidence of edema (Fig. 1, $A$ ).

Vascular congestion. Mild or moderate parenchymal vascular congestion (Fig. $1, B$ ) was seen in two nontransplanted samples after 48 hours of storage and in four after 72 hours of storage. All 

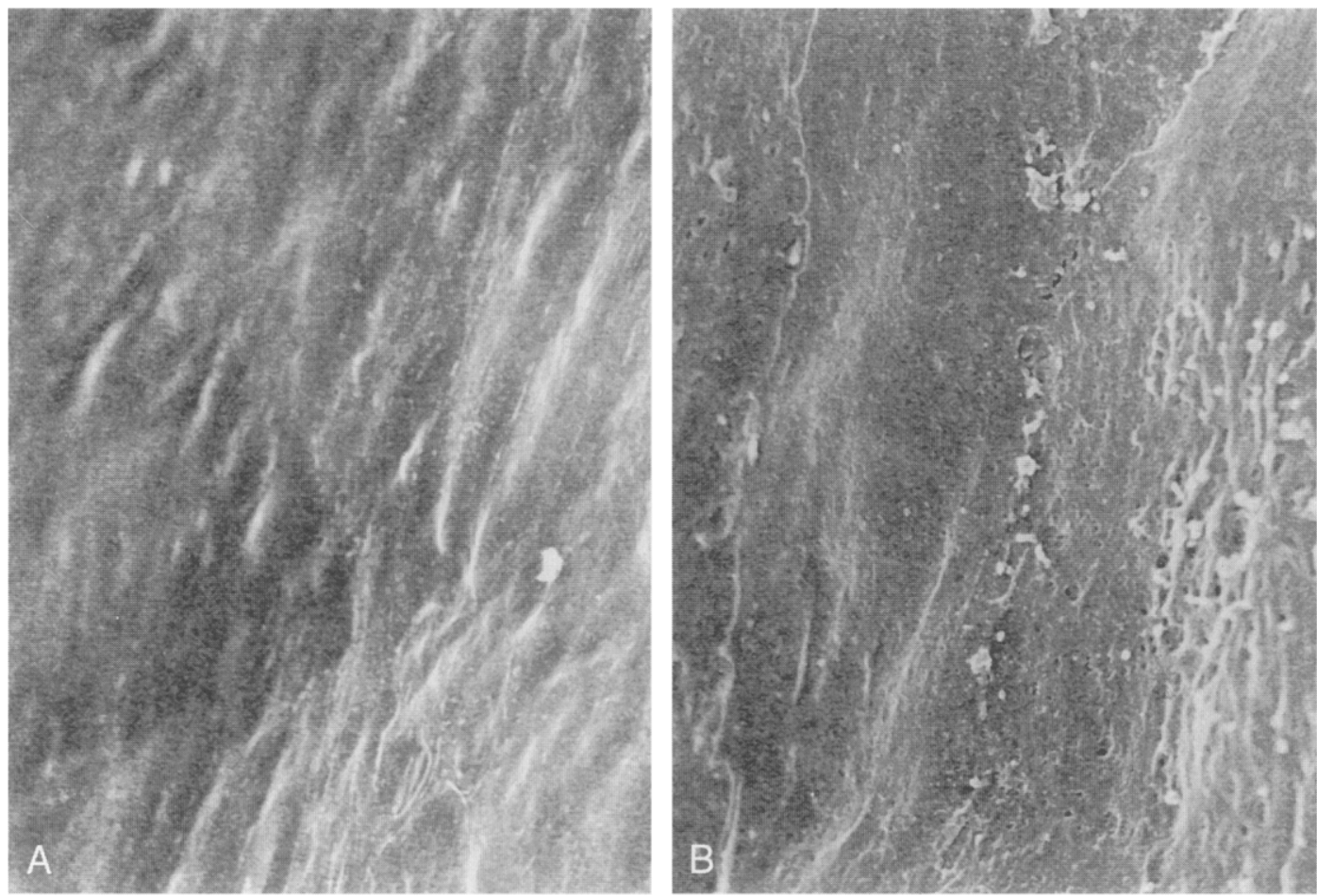

Fig. 4. Electron micrographs of normal rat lung endothelium. The whole of the surface was covered with regularly shaped ECs in close contact with each other. (Original magnifications: $\mathbf{A}, \times 1600 ; \mathbf{B}, \times 8000$.)

transplanted samples showed vascular congestion. This ranged from moderate to severe in groups $3 \mathrm{a}$ and $3 \mathrm{~b}$. Congestion ranged from mild to severe in group $4 \mathrm{a}$, whereas severe congestion was present in all cases from group $4 \mathrm{~b}$. There was, however, no statistically significant difference among the different groups for this parameter.

Perivascular edema and hemorrhage. Perivascular edema (Fig. 2, $A$ ) was identified in all of the nontransplanted and transplanted samples included in the study. In the nontransplanted lungs, edema consisted of acellular fluid. After reperfusion, perivascular hemorrhage was also identified. Hemorrhage was present in one specimen $(n=7)$ after 72 hours of storage and 5 minutes of revascularization, in five of seven specimens after 48 hours of storage and 24 hours of revascularization, and in all seven specimens after 72 hours of storage and 24 hours of reperfusion.

Alveolar edema and hemorrhage. None of the stored, nontransplanted samples showed alveolar edema or hemorrhage. After 5 minutes of reperfusion, no hemorrhage was present in any sample. However, edema (Fig. 1, $B$ ) was seen in three of seven specimens each from the 48-hour storage period (two cases mild, one moderate) and the 72-hour storage period (minor in one case and mild in two). After 24 hours of reperfusion, edema and hemorrhage (Fig. 2, B) were seen in all samples from both storage times. There was a statistically significant positive correlation between the different revascularization times for both alveolar edema $(p<0.001)$ and hemorrhage $(p<0.001)$.

Bronchial epithelial changes. Bronchial alterations consisted of loss of cilia, blebbing of the apical cytoplasm, increased eosinophilia of the cells, rounding up of cell shape, and detachment of blebs and individual cells (Fig. 3, $A$ ). These changes were seen in all samples from both storage times and after 0 and 5 of minutes reperfusion. The changes ranged from mild to severe. In samples stored for 48 hours, bronchial changes had been totally reversed after 24 hours of reperfusion (Fig. 3, $B$ ) with only mild changes present in one of seven specimens. Bronchial changes could not be assessed in any 72 -hour stored specimens after 24 hours of reperfusion because more than $80 \%$ of the bronchial epithelium was denuded. All these specimens had severe alveolar hemorrhage with features of infarction in some (three of seven). There was a statistically significant 

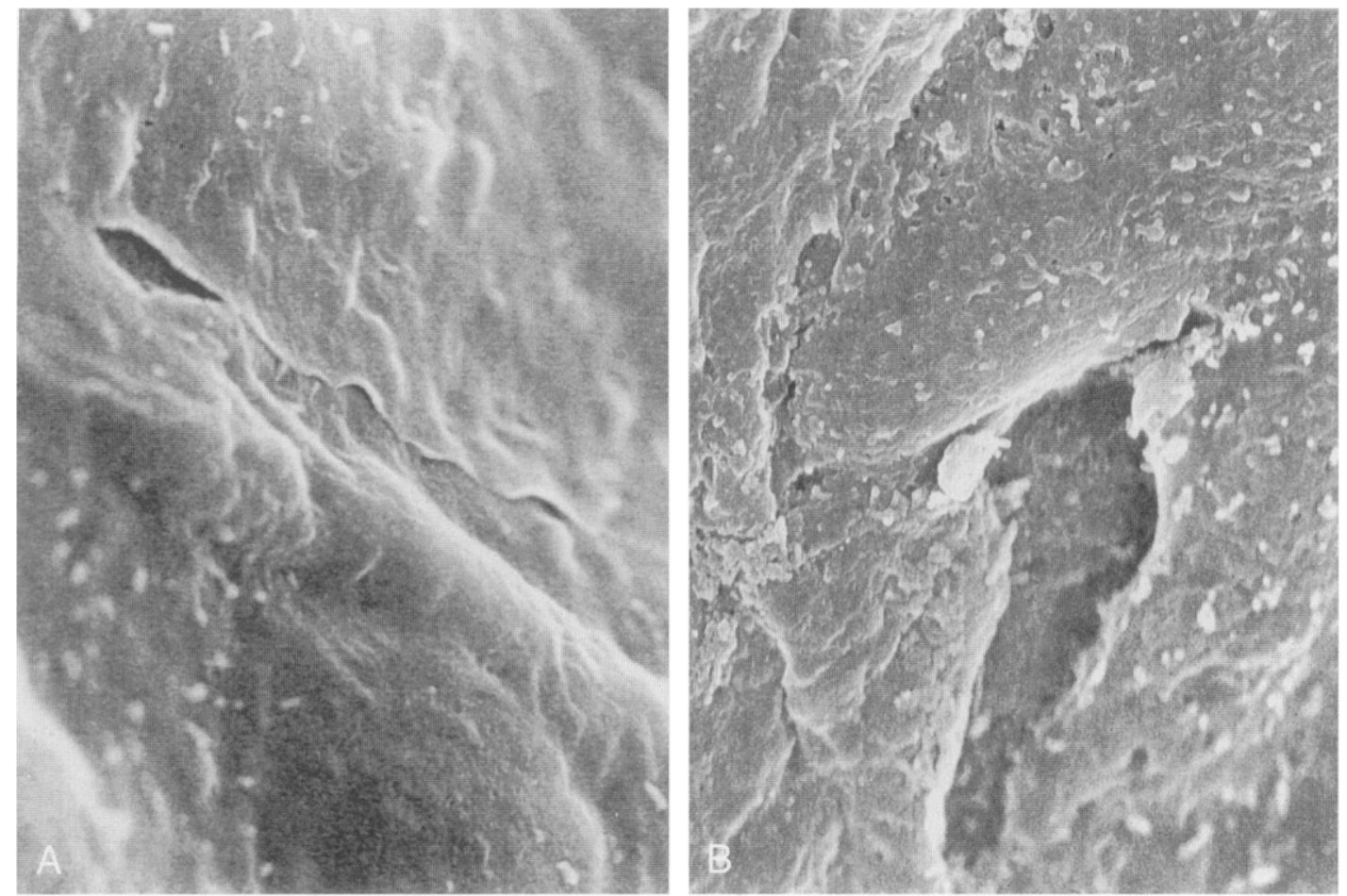

Fig. 5. Electron micrographs of rat pulmonary arterial endothelium. A, Storage for 48 hours; B, storage for 72 hours and revascularization for 5 minutes. A small intercellular gap can be seen between two ECs (A), and a semidetached cell (B) exposes basal matrix. (Original magnifications $\times 8000$.)

negative correlation of the bronchial epithelial changes with the different revascularization periods $(p<0.01)$.

SEM analysis. Control specimens presented a uniform monolayer of regular ECs in all micrographs. The entire surface was covered by fusiformshaped ECs in close contact with each other (Fig. 4, A). A small number of microvilli were identified on the surface of ECs observed at high magnification (Fig. 4, $B$ ).

Stored but nonrevascularized lungs (group 2) had continuous monolayers with few variations from control specimens. Some monolayers, especially in the 72-hour stored group, had small intercellular gaps (Fig. 5, $A$ ).

All stored lungs subjected to 5 minutes of revascularization (group 3) had some degree of damage. Some areas appeared denuded of ECs with exposure of basal matrix and collagen fibers, which was more marked in the 72-hour stored group. Other ECs had already lost intercellular connections with neighboring cells (Fig. 5, B). Increased numbers and size of surface microvilli and presence of membrane blebs were common in specimens in this group (Fig. 6, $A$ ).

Stored lungs subjected to 24 hours of revascularization (group 4) had patches of well-preserved endothelium coexisting with denuded areas where blood clot and blood cells obscured the endothelial surface. Some specimens had retracted ECs with delicate intercellular connections and numerous intercellular gaps (Fig. 6, B). These monolayers appeared to have little or no control of vascular permeability despite the presence of a noncontinuous monolayer of ECs. The changes were again more marked in the 72-hour stored group.

Because of the small number of specimens in each group, statistical analysis comparing specimens stored for 48 hours versus specimens stored for 72 hours failed to find significant differences in endothelial preservation; similarly, no significant correlation between storage and revascularization period was found, although a trend was seen suggesting that specimens stored for 72 hours were more damaged than specimens stored for 48 hours, and that damage was exacerbated by revascularization. 

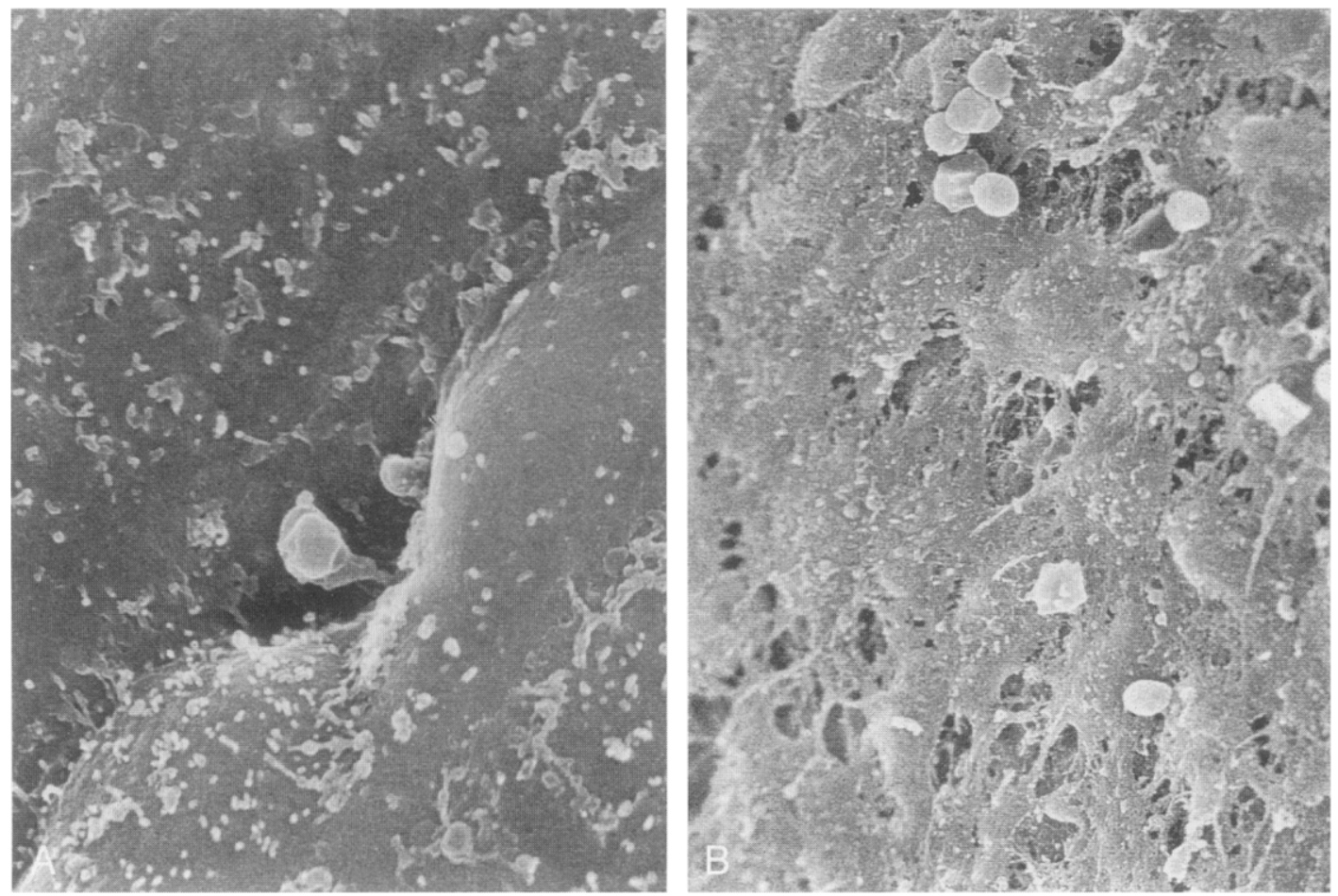

Fig. 6. Electron micrographs of rat pulmonary arterial endothelium stored for 48 hours, followed by 5 minutes (A) or 24 hours (B) of revascularization. An increased number of surface microvilli and membrane blebs can be identified on EC surfaces at high magnification (A, original magnification $\times 8000$ ). Discontinuities and loose intercellular connections can be appreciated at lower magnification (B, original magnification $\times 1600)$.

\section{Discussion}

A connection between vascular endothelial damage during preservation and parenchymal edema in transplanted lungs has long been suspected. Under physiologic conditions the endothelial monolayer plays a crucial role in regulating vascular permeability. Passage of fluids and macromolecules to the parenchymal space, as well as inflammatory cell migration and other functions involving transfer of cellular elements from the vascular to the alveolar space, are directly or indirectly regulated by a single layer of ECs. ${ }^{15}$

After ischemia and reperfusion, stored lungs lose control of vascular permeability, and edema develops., 16, 17 In vitro studies have also shown that hypoxia and reoxygenation increase the permeability of cultured EC monolayers. ${ }^{18}$ However, to our knowledge, direct morphologic evidence linking endothelial monolayer damage to alveolar edema in transplanted lungs has not been reported before. The present results show a clear correlation between the degrees of alteration in pulmonary endothelial monolayer continuity, EC shape and attachment, and the amount of parenchymal edema found in lungs undergoing transplantation.

Perivascular edema was found in all stored lungs before revascularization. The source of edema during the preservation period is likely to be the preservation solution itself, as was suggested by Hall and coworkers. ${ }^{16}$ This edema could reach the perivascular interstitial tissue via small gaps seen in the previously confluent monolayer, which are probably caused by weakening of intercellular connections between adjacent ECs. Alveolar edema was identified after 5 minutes of revascularization, but hemorrhage appeared only 24 hours after transplantation, when the observed damage to endothelium was greater. In these lungs, especially after 72 hours of hypothermic storage, blood cells were able to reach the alveoli and all specimens showed perivascular hemorrhage. Correlation between alveolar hemorrhage and revascularization time reached statistical significance $(p<0.001)$.

Several mechanisms of damage to ECs possibly 
interact under the conditions studied here. First, severe hypothermia for a prolonged period is harmful to EC structure and viability in vitro. ${ }^{19,20}$ Falling levels of cellular adenosine triphosphate have been suggested as a cause for the cytoskeletal disassembly that takes place during hypothermic storage of ECs. ${ }^{21}$ Second, hypoxia alone has been shown to be highly damaging to pulmonary ECs by a combination of stimulation of membrane lipid peroxidation and degradation of membrane phospholipids. ${ }^{22}$ The association of ischemia and hypothermia results in severe stress for ECs. Experiments with cultured human ECs in our laboratory showed marked morphologic alterations of monolayers subjected to cold hypoxic storage. ${ }^{23}$ Liver transplantation studies found sinusoidal ECs to be more sensitive to ischemic damage than parenchymal cells. ${ }^{11}$ Third, revascularization involves the sudden restoration of highly oxygenated blood at normal arterial pressure. SEM studies do not allow measurement of the strength of EC attachment to the basal matrix or to each other via intercellular connections. The retraction of ECs, together with the change of shape (rounding) seen in the present study and in other ultrastructural studies of endothelium, allows gap formation in the monolayer to be recorded; however, the possibility of normal-shaped but weakly anchored ECs cannot be ruled out. Consequently, small morphologic changes in ECs before revascularization could be accompanied by a more extreme denudation after pulmonary circulation is restored. Finally, additional damage is likely to be caused by oxygen-derived free radicals, known to increase permeability of EC monolayers in vitro after ischemia and reperfusion, probably by cell retraction. ${ }^{18}$

Another serious pathologic event frequently found after organ storage in clinical practice is the increased thrombogenicity of the vascular bed in the transplanted organ. ${ }^{24}$ The exposure of thrombogenic basal matrix and collagen elements after storage and revascularization seen in the present experiment could explain those alterations. Also, ECs have been shown to switch from anticoagulant to procoagulant properties when subjected to hypoxic conditions. ${ }^{25}$ According to the evidence presented in this study, denudation and exposure of thrombogenic basal matrix is likely to be a main cause of coagulation problems after long periods of hypothermic storage.

EC surface blebbing has been described previously in hypoxic cells. Ultrastructural studies with liver sinusoidal ECs showed that blebbing is a common feature of injured cells. ${ }^{26}$ Previous SEM studies allowed us to conclude that blebs originated from swollen microvilli, which detached from the cell surface and became spherical free bodies ${ }^{27}$; this observation is in accordance with information reported by Lemasters and coworkers, ${ }^{28}$ who collected detached blebs by filtration of effluent perfusate of isolated rat liver during hypoxia and reoxygenation processes. Blebbing seems to be a step before cell death and could be a defense mechanism in volumecompromised cells because of the hypothermic inactivation of the sodium-potassium membrane pump. Blebbing of parenchymal cells resulting from ischemic-hypothermic damage has been described in hepatocytes of livers being transplanted. ${ }^{29}$

An unexpected histologic finding in the present experiment was the alteration of bronchial epithelial cells as a consequence of cold hypoxic storage. We found cell rounding and detachment, ciliary alterations, and blebbing. These bronchial epithelial changes may be a result of hypoxia and hypothermia during storage. After reperfusion, however, the changes regressed in lungs stored for 48 hours, suggesting that these alterations were reversible. In lungs stored for 72 hours, however, denudation of the epithelium was associated with marked parenchymal hemorrhage, and in some specimens with infarction.

These two changes (rounding and blebbing) were common to ECs and bronchial epithelial cells in the present study. The tendency of these two types of cells to become spherical leads us to speculate about the existence of a mechanism toward cell rounding and loss of physiologic cell shape under hypoxic and hypothermic conditions that could be universal, dependent more on the space available to become round than on the shape adopted by the different cells. Luminal cells (vascular ECs and bronchial epithelial cells in this study) have the space to become spherical, whereas most other parenchymal cells are in three-dimensional close contact with each other, which renders shape changes difficult. As mentioned earlier, blebbing is also present in other parenchymal cells subjected to ischemic-hypothermic damage.

We conclude that perivascular and alveolar edema found in preserved lungs was caused by morphologic alterations of ECs resulting in monolayer discontinuities followed by cell detachment. These alterations were present after the storage period and became more marked with the restoration of blood flow on revascularization. Morpho- 
logic alteration and cytoplasmic blebbing of ECs (SEM) and bronchial epithelial cells (histology) were also a consequence of the cold hypoxic storage period.

We thank Caroline Doré, BSc, for her help with the statistical analysis.

\section{REFERENCES}

1. Orak JK, Singh AK, Rajagopalan PR, Singh I. Morphological analysis of mitochondrial integrity in prolonged cold renal ischemia utilizing Euro-Collins versus University of Wisconsin preservation solution in a whole organ model. Transplant Proc 1994;26:122-5.

2. Toledo-Pereyra LH, Rodríguez FJ. Scientific basis and current status of organ preservation. Transplant Proc 1994;26: 309-11.

3. Novick RJ, Menkis AH, McKenzie FN. New trends in lung preservation: a collective review. J Heart Lung Transplant 1992;11:377-92.

4. Hidalgo MA, Manek S, Fryer PR, Fuller BJ, Green CJ. Morphological changes in rat single lung isografts after long-term survival. Int J Exp Pathol 1995;76:43-54.

5. Kurzawinski TR, Applebly JA, Hardy SC, et al. A prospective, randomised clinical trial of liver preservation using high-sodium versus high-potassium lactobionate/raffinose solution. Transplant Int 1994;7:S489-92.

6. Hamvas A, Palazzo R, Kaiser L, et al. Inflammation and oxygen free radical formation during pulmonary ischemiareperfusion injury. J Appl Physiol 1992;72:621-8.

7. Naka Y, Shirakura R, Matsuda H, et al. Canine heart-lung transplantation after twenty-four-hour hypothermic preservation with Belzer-UW solution. $\mathbf{J}$ Heart Lung Tranplant 1991;10:296-303.

8. Zilla $P$, von Oppell $U$, Deutsch $M$. The endothelium: a key to the future. J Card Surg 1993;8:32-60.

9. Taylor AE. Pulmonary edema: ischemia reperfusion endothelial injury and its reversal by c-AMP ${ }^{+}$. Proc Natl Sci Counc Repub China 1991;15:191-5.

10. Caldwell-Kenkel JC, Currin RT, Tanaka Y, Thurman RG, Lemasters JJ. Reperfusion injury to endothelial cells following cold ischemic storage of rat livers. Hepatology 1989;10: 292-9.

11. McKeown CMB, Edwards V, Phillips MJ, Harvey PRC, Petrunka CN, Strasberg SM. Sinusoidal lining cell damage: the critical injury in cold preservation of liver allografts in the rat. Transplantation 1988;46:178-91.

12. Malik AB, Lynch JJ, Cooper JA. Endothelial barrier function. J Invest Dermatol 1989;93:62S-7S.

13. Grosso MA, Brown JM, Viders DE, et al. Xanthine oxidasederived oxygen radicals induce pulmonary edema via direct endothelial cell injury. J Surg Res 1989;46:355-60.

14. Jamieson NV, Sundberg R, Lindell S, et al. Preservation of the canine liver for 34-48 hours using simple cold storage with UW solution. Transplantation 1988;46:517-22.
15. Hinsbergh van VWM. Regulatory functions of the coronary endothelium. Mol Cell Biochem 1992;116:163-9.

16. Hall SM, Odom N, McGregor CGA, Haworth SG. Transient ultrastructural injury and repair of pulmonary capillaries in transplanted rat lung: effect of preservation and reperfusion. Am J Respir Cell Mol Biol 1992;7:49-57.

17. Mills AN, Hooper TL, Hall SM, McGregor CGA, Haworth SG. Unilateral lung transplantation: ultrastructural studies of ischemia-reperfusion injury and repair in the canine pulmonary vasculature. J Heart Lung Transplant 1992;11: $58-67$.

18. Inauen W, Payne DK, Kvietys PR, Granger DN, Hypoxiareoxygenation increases the permeability of endothelial cell monolayers: role of oxygen radicals. Free Radic Biol Med 1990;9:219-23.

19. Solberg S, Larsen T, Jørgensen L, Sørlie D. Cold induced endothelial cell detachment in human saphenous vein grafts. J Cardiovasc Surg 1987;28:571-5.

20. Hidalgo MA, Ambrose IJ, Goddard JG, Fuller BJ, Green CJ. Cultured human endothelial cells as a model for hypothermic preservation. Cryoletters 1993;14:339-46.

21. Hinshaw DB, Burger JM, Armstrong BC, Hyslop PA. Mechanism of endothelial cell shape change in oxidant injury. J Surg Res 1989;46:339-49.

22. Block ER, Patel JM, Edwards D. Mechanism of hypoxic injury to pulmonary artery endothelial cell plasma membranes. Am J Physiol 1989;257:C223-31.

23. Hidalgo MA, Mann DJ, Thorniley MS, Fuller BJ, Green CJ. Cytoskeleton oriented preservation solution protects endothelial cells monolayer against cold ischaemia. Clin Sci $1994 ; 87: 26$.

24. Arai M, Mochida S, Ohno A, Kurokawa K, Fujiwara K. Coagulability in the sinusoids of orthotopically transplanted livers in rats. Transplant Proc 1994;26:913-5.

25. Gertler JP, Weibe DA, Ocasio VH, Abbott WM. Hypoxia induces procoagulant activity in cultured human venous endothelium. J Vasc Surg 1991;13:428-33.

26. Okouchi Y, Sasaki K, Tamaki T. Ultrastructural changes in hepatocytes, sinusoidal endothelial cells and macrophages in hypothermic preservation of the rat liver with University of Wisconsin solution. Virchows Arch 1994;424:477-84.

27. Hidalgo MA, Sarathchandra P, Fryer PR, Fuller BJ, Green CJ. Scanning electron microscopic changes in morphology of pulmonary endothelium in rat lung isografts following hypothermic ischaemic storage and transplantation. Int $\mathrm{J}$ Exp Pathol 1995;76:339-51.

28. Lemasters JJ, Stemkowski CJ, Ji S, Thurman RG. Cell surface changes and enzyme release during hypoxia and reoxygenation in the isolated, perfused rat liver. $\mathbf{J}$ Cell Biol 1983;97:778-86.

29. Carles J, Fawaz R, Hamoudi NE, Neaud V, Balabaud C, Bioulac-Sage P. Preservation of human liver grafts in UW solution: ultrastructural evidence for endothelial and Kupffer cell activation cold ischaemia and after ischaemia-reperfusion. Liver 1994;14:50-6. 